\title{
The Dynamical Equilibrium of Galaxy Clusters
}

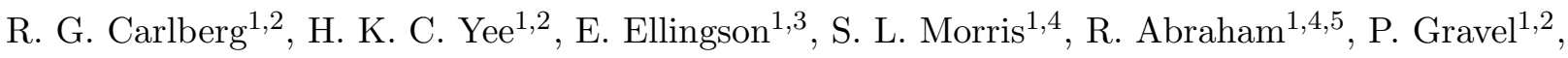 \\ C. J. Pritchet ${ }^{1,6}$, T. Smecker-Hane ${ }^{1,4,7}$ F. D. A. Hartwick ${ }^{6}$ J. E. Hesser ${ }^{4}$, J. B. Hutchings ${ }^{4}, \&$ \\ J. B. Oke ${ }^{4}$
}

\begin{abstract}
If a galaxy cluster is effectively in dynamical equilibrium then all galaxy populations within the cluster must have distributions in velocity and position that individually reflect the same underlying mass distribution, although the derived virial masses can be quite different. Specifically, within the CNOC cluster sample the virial radius of the red galaxy population is, on the average, a factor of $2.05 \pm 0.34$ smaller than that of the blue population. The red galaxies also have a smaller RMS velocity dispersion, a factor of $1.31 \pm 0.13$ within our sample. Consequently, the virial mass calculated from the blue galaxies is $3.5 \pm 1.3$ times larger than from the red galaxies. However, applying the Jeans equation of stellar-hydrodynamical equilibrium to the red and blue subsamples separately give statistically identical cluster mass profiles. This is strong evidence that these clusters are effectively equilibrium systems, and therefore empirically demonstrates that the masses in the virialized region are reliably estimated using dynamical techniques.
\end{abstract}

Subject headings: galaxies: clusters, cosmology: large-scale structure of universe

\section{Introduction}

The primary goal of the CNOC (Canadian Network for Observational Cosmology) cluster redshift survey is to obtain a value of density parameter, $\Omega_{0}$, for those components of the mass

\footnotetext{
${ }^{1}$ Visiting Astronomer, Canada-France-Hawaii Telescope, which is operated by the National Research Council of Canada, le Centre National de Recherche Scientifique, and the University of Hawaii.

${ }^{2}$ Department of Astronomy, University of Toronto, Toronto ON, M5S 3H8 Canada

${ }^{3}$ Center for Astrophysics \& Space Astronomy, University of Colorado, CO 80309, USA

${ }^{4}$ Dominion Astrophysical Observatory, Herzberg Institute of Astrophysics, National Research Council of Canada, 5071 West Saanich Road, Victoria, BC, V8X 4M6, Canada

${ }^{5}$ Institute of Astronomy, Madingley Road, Cambridge CB3 OHA, UK

${ }^{6}$ Department of Physics \& Astronomy, University of Victoria, Victoria, BC, V8W 3P6, Canada

${ }^{7}$ Department of Physics \& Astronomy, University of California, Irvine, CA 92717, USA
} 
field (possibly all) that participate in gravitational clustering (Carlberg et al. 1996, hereafter CNOCi). The product of the field luminosity density, $j$, with its mass-to-light ratio, $M / L$, estimates the mean mass density of the universe, $\rho_{0}$ (Oort 1958), which in ratio to the critical density, $\rho_{c}$ is equal to $\Omega_{0}$ (Gott \& Turner 1976), when calculated in current epoch co-ordinates. The crucial quantity is an estimator of the field mass-to-light ratio, for which we use the cluster virial mass-to-light ratio, $M_{v} / L$. The main technical concern of the CNOC survey is that there are various possibilities that $M_{v}$ is a biased estimator of the cluster mass. Besides statistical biases, dissipative processes can cause the cluster galaxies to become more centrally concentrated than the cluster mass. In addition, when estimating the field $M / L$ from the cluster results, allowance must be made for the differences between the cluster and field galaxy populations.

To address these issues a sample of high X-ray luminosity galaxy clusters were selected from the EMSS catalogue (Henry et al. 1992, Gioia \& Luppino 1994). This produces a relatively homogenous set of 16 clusters that likely contain a substantial virialized component. The sample, at a mean redshift of about $1 / 3$, gives a substantial column in redshift space which we use to calculate the field luminosity density in precisely the same measurement system as the cluster light. The galaxies were selected with no knowledge of the colour or whether they were cluster members, although great care is taken to control the selection effects that are unavoidable to maintain efficiency (Yee, Ellingson \& Carlberg 1996).

From these data we calculated the line-of-sight velocity dispersions, $\sigma_{1}$, and virial radii, $r_{v}$, of the clusters to derive the virial masses (Binney \& Tremaine 1987), $G M_{v}=3 \sigma_{1}^{2} r_{v}$. The velocity dispersions are calculated with an iterative technique that statistically removes interlopers by taking advantage of the relatively large foreground and background sample to estimate interloper densities (CNOCi $)$. This technique leads to velocity dispersions that are on the average $7 \%$ less than those found from precisely the same data in the redshift range, and about $13 \%$ less with a somewhat less restrictive redshift cut, even when evaluated with the iterated bi-weight estimator (Beers, Flynn \& Gebhardt 1990). The virial radius is calculated from the data in the redshift range of the cluster with a "ring-wise" potential estimator, which allows correction for the strip sampling and helps reduce the noise (details are given in CNOCi $)$. All these global quantities are derived from the individual galaxy positions, velocities, colors and luminosities, for which objective errors are calculated using the Jackknife method (Efron \& Tibshirani 1986). The errors are sufficiently small that the assumption of a symmetric distribution is appropriate and is verified by comparing to Bootstrap error estimates.

The average cluster virial mass-to-light ( $r$ band, k-corrected) ratio is $\left\langle M_{v} / L_{r}^{k}\right\rangle=$ $289 \pm 50 h \mathrm{M}_{\odot} / \mathrm{L}_{\odot}$ (CNOCi). In the field, over the same redshift range, with the same sampling and corrections, the closure mass-to-light ratio is $\rho_{c} / j=1136 \pm 138 h \mathrm{M}_{\odot} / \mathrm{L}_{\odot}$ (in co-moving units, for $\left.q_{0}=0.1\right)$. There are two additional corrections applied to $\left\langle M_{v} / L_{r}^{k}\right\rangle$. The mean luminosity of galaxies brighter than $M_{r}^{k}$ of $-18.5 \mathrm{mag}$ is $0.11 \pm 0.05$ lower in the cluster than the field and the analysis of the mass profile (Carlberg, Yee \& Ellingson 1997, hereafter CNOCii) showed that $M_{v}$ needed to be multiplied by $0.82 \pm 0.14$, which we attribute to neglecting the surface term in the 
virial mass estimator. Making these corrections leads to a redshift adjusted $\Omega_{0}=0.19 \pm 0.06$.

Our conclusion that the stellar-hydrodynamical (SHD) mass profile of the clusters averaged together closely follows the number density profile of the galaxies (CNOCii) rests on the assumption that the clusters are effectively in equilibrium such that the Jeans equation can be used to derive the mass profile from the radial and velocity distribution of any tracer population. In this paper we demonstrate that two radically different tracer populations are consistent with the same underlying mass profile, which we take as strong support of the equilibrium assumption. In the following section we describe the division of the sample into two completely disjoint blue and red subsamples, whose surface density and projected velocity dispersion profiles are measured and demonstrate the gross differences in the calculated virial masses. In Section 3 we derive the stellar-hydrodynamical potential generating mass profiles in which the galaxies are orbiting. The implications of the statistical equality of the two profiles are discussed in the final section. All calculations in this paper assume $H_{0}=100 \mathrm{~km} \mathrm{~s}^{-1} \mathrm{Mpc}^{-1}$ and $q_{0}=0.1$, although the results are either independent or not sensitive to these choices.

\section{The Blue and Red Subsamples}

The CNOC cluster redshift survey catalogues (Yee, Ellingson \& Carlberg 1996) contain $~ 2600$ galaxies having Gunn $r$ magnitudes, $g-r$ colors, and redshifts. The sample is split at the color $(g-r)_{z}=(g-r)[1.2+2.33(z-0.3)]^{-1}$ of 0.7 (CNOCi) . This is approximately the color of an Sab galaxy, which roughly divides galaxies into spheroid dominated, "old" stellar systems and disk dominated, "young" stellar systems. Fourteen clusters are averaged together after removing two "binary" clusters.

To a limit of $M_{r}^{k}=-18.5 \mathrm{mag}$, about $70 \%$ of the cluster galaxies fall into the red subsample. The methods of analysis are precisely the same as used previously for the full sample (CNOCii). The velocities are normalized to the $\sigma_{1}$ of each cluster calculated from galaxies of all colors so that the two subsamples have a common reference. The RMS velocity dispersions of the red and blue galaxies are $0.97 \pm 0.04$ and $1.27 \pm 0.11$, respectively, in units where the velocities are normalized to the velocity dispersions of all the galaxies in each cluster. That is, the velocity dispersion of blue cluster galaxies is about 30\% higher than for red galaxies (Rood et al. 1972, Kent \& Gunn 1982, Stein 1996). The projected radii are normalized to $r_{200}=\sqrt{3} \sigma_{1} /(10 H(z))$, the radius at which the mean interior overdensity in a cluster is $200 \rho_{c}(z)$. The quantity $H(z)$ is the Hubble constant at the redshift of the cluster. The virial radii, $r_{v}$, are $2.58 \pm 0.27$ and $5.28 \pm 0.73$ for red and blue galaxies, respectively, in $r_{200}$ units. In ratio to the sample as a whole, the average virial masses are $0.66 \pm 0.08$ and $2.31 \pm 0.42$, respectively, for red and blue subsamples. The ratio of these two virial masses is $3.5 \pm 1.3$. As is well known, the red galaxies in clusters are much more centrally concentrated than the blue galaxies (e.g. Dressler 1980). Our data usefully illustrate that the galaxy color selection can make a very large difference to the estimated mass. In general, blue or emission line selected galaxies will always give higher mass estimates than absorption line 
galaxies.

The projected surface number density profiles, $\Sigma_{N}(R)$ of the two subsamples are displayed in Figure 1. The profiles are fitted to the projection of the density function (Hernquist 1990),

$$
\nu(r)=\frac{A}{r(r+a)^{3}},
$$

by adjusting $A$ and $a$ to minimize the residuals. The fits give scale radii $a$ of $0.56 \pm 0.10$ and $1.82 \pm 0.27$ for red and blue populations, respectively. For the sample as a whole $a=0.66 \pm 0.09$. In both cases the $\chi^{2}$ per degree of freedom is about 0.8 , with the errors estimated as the average of the upper and lower $1 \sigma$ confidence range from the bootstrap analysis.

The observed projected velocity dispersion, $\sigma_{p}(R)$, can be used to infer the real space radial velocity dispersion, $\sigma_{r}(r)$, via,

$$
\sigma_{p}^{2}(R) \Sigma_{N}(R)=\int_{R}^{\infty} \nu(r) \sigma_{r}^{2}\left(1-\beta \frac{R^{2}}{r^{2}}\right) \frac{r}{\sqrt{r^{2}-R^{2}}} d r
$$

where $\beta=1-\sigma_{\theta}^{2} / \sigma_{r}^{2}$ is the velocity anisotropy parameter. The parameters of

$$
\sigma_{r}^{2}(r)=\frac{B}{b+r}
$$

are adjusted until the $\chi^{2}$ with the observed $\sigma_{p}(R)$ are minimized. The data and the fits for two values of $\beta$ are displayed in Figure 2. Again the errors are from the symmetrized bootstrap confidence range.

\section{The Mass Distribution}

The mass distribution in which the galaxies are in orbital equilibrium can be inferred with no assumption about the relative distribution of mass and galaxies from the projected velocity dispersion profile, $\sigma_{p}(R)$, and the projected galaxy density distribution, $\Sigma_{N}(R)$. Remarkably, we found (CNOCii) that the integrated galaxy number density profile, $L(r)$, of our full sample is statistically indistinguishable from the integrated mass profile, $M(r)$.

Mass profiles are derived from the fitted $\nu(r)$ and $\sigma_{r}(r)$ using the Jeans equation Binney \& Tremaine 1987),

$$
M_{S H D}(r)=-\frac{\sigma_{r}^{2} r}{G}\left[\frac{d \ln \sigma_{r}^{2}}{d \ln r}+\frac{d \ln \nu}{d \ln r}+2 \beta\right] .
$$

We will call this mass the stellar hydrodynamical mass. The resulting $M_{S H D}(r)$ from the red and blue galaxies are shown in Figure 3 for $\beta=0$ and $1 / 2$. The quantity plotted is the virial mass-to-light bias,

$$
b_{M v}(r)=\frac{M_{S H D}(r)}{L(r)} \frac{\tilde{L}}{\tilde{M}_{v}},
$$


where $\tilde{L}$ is an arbitrary normalization of the total light that cancels between numerator and denominator. The quantity $\tilde{M}_{v}$ is the normalization of the virial mass of the dimensionless sample as a whole, $\tilde{M}_{v}=3{\tilde{\sigma_{1}}}^{2} \tilde{r}_{v}$, where $\tilde{\sigma_{1}}=1.05 \pm 0.04$ and $\tilde{r}_{v}=3.35 \pm 0.32$. If the virial mass calculated from the sample as a whole gave the correct $M / L$ at all radii then $b_{M v}(r)=1$ everywhere. If the tracer population follows the true mass profile but with some scale error, then $b_{M v}$ will be a constant other than unity. Figure 3 shows that beyond about $0.3 r_{200}$ the mass profiles deduced from the two subsamples are identical within their errors (about 15 and 30\% for red and blue mass profiles, respectively). Figure 4 shows the value of $b_{M v}$ evaluated at $r_{200}$, demonstrating that the blue and red subsamples give statistically identical values. Furthermore the value is always less than unity, as we found for the sample as a whole (CNOCii) which has the implication that although the galaxy numbers fairly accurately trace the cluster mass profile the virial mass to light ratio is always an overestimate of the mean $M / L$ inside $r_{200}$. There is no statistically significant radial gradient of $b_{M v}(r)$ for $r \simeq r_{200}$.

There are several important conclusions to be drawn from Figure 3. First, it provides strong evidence that the blue and red galaxies are sufficiently in equilibrium with the cluster mass distribution that these dynamical methods work to recover the true mass profile. Second, the fitted scale radius of the mass profile (taken to be equal to that of the full sample) $a=0.66 \pm 0.09$, is slightly more extended than the red galaxies (Tyson \& Fischer 1995) $a=0.56 \pm 0.10$, but is substantially more compact than the blue galaxy distribution, $a=1.82 \pm 0.27$, all measured in $r_{200}$ units. The differences in scale radii imply that neither the blue nor the red galaxies are distributed like the mass, so the virial masses calculated from these subsamples are not correct.

\section{Conclusions}

The densities and velocity dispersions of the red and blue galaxy subsamples, although very different from each other, imply a statistically identical mass profile for the cluster potential. This is necessary if both populations are in equilibrium with the potential, validating our use of the Jeans equation. This mass profile is statistically identical to the number density distribution of all the galaxies of our full sample. The limiting factor in the precise numerical agreement is that there are relatively few blue galaxies in clusters, meaning that their density and velocity dispersion profiles are less accurately measured.

There are problems and issues that cannot be addressed with a sample of this size. A much larger sample would likely find that a common value of $\beta$ would not work for both red and blue galaxies, since it is expected that on the average blue galaxies will avoid the core, hence near the centre have smaller $\beta$ than the red galaxies, and at large radius include many objects on their first cluster crossing, hence have higher $\beta$ than the red galaxies. Furthermore the galaxies themselves are not invariant mass points. The blue galaxies are being altered by the cluster environment such that some of the their members are likely leaving the blue population to join the red population Abraham et al. 1996). 
The virial mass calculated from the full sample, empirically adjusted for its measurement biases, will correctly estimate the mass enclosed. With these two subsamples we have now tested each step in the chain of logic which supports our corrected, population adjusted value of $\Omega_{0}=0.19 \pm 0.06$. There is no compelling evidence for any remaining systematic errors of the cluster $M / L$ as an estimator of the field value over the redshift range of this sample.

We thank the Canadian Time Assignment Committee of the CFHT for allocations of observing time, and the CFHT organization for the technical support which made these observations feasible. Funding was provided by NSERC and NRC of Canada. 


\section{REFERENCES}

Abraham, R. G., Smecker-Hane, T. A., Hutchings, J. B., Carlberg, R. G., Yee, H. K. C., Ellingson, E., Morris, S., Oke, J. B., Davidge, T. 1996, ApJ, 471, 694

Beers, T. C., Flynn, K. \& Gebhardt, K. 1990, AJ, 100, 32

Binney, J. \& Tremaine, S. 1987, Galactic Dynamics, (Princeton University Press: Princeton)

Carlberg, R. G., Yee, H. K. C., Ellingson, E., Abraham, R., Gravel, P., Morris, S. M, \& Pritchet, C. J. 1996 (CNOCi), ApJ, 462, 32

Carlberg, R. G., Yee, H. K. C., \& Ellingson, E., 1997 (CNOCii), ApJ, in press

Dressler, A. 1980, ApJ, 236, 351

Efron, B. \& Tibshirani, R. 1986, Statistical Science, 1, 54

Gioia, I. M. \& Luppino, G. A. 1994, ApJS, 94, 583

Gott, J. R. \& Turner, E. L 1976, ApJ, 209, 1

Henry, J. P., Gioia, I. M., Maccacaro, T., Morris, S. L., Stocke, J. T., \& Wolter, A. 1992, ApJ, 386,408

Hernquist, L. 1990, ApJ, 356, 359

Kent, S. \& Gunn, J. E. 1982, AJ, 87, 945

Oort, J. H. 1958, in La Structure et L'Évolution de L'Univers, Onzième Conseil de Physique, ed. R. Stoops (Solvay Institute: Bruxelles) p. 163

Rood, H. J., Page, T. L, Kintner, E. C. \& King, I. R. 1972, ApJ, 175, 627

Stein, P. 1996, A\&A, in press (astroph/9606162)

Tyson, J. A. \& Fischer, P. 1995, ApJ, 446, L55

Yee, H. K. C., Ellingson, E. \& Carlberg, R. G. 1996, ApJS, 102, 269 
Fig. 1. - The projected number density profiles of the blue (solid squares and dotted lines) and red (circles and solid lines) cluster galaxies. The $1 \sigma$ confidence range from a Bootstrap error estimate is shown.

Fig. 2.- The RMS line-of-sight velocity dispersion, $\sigma_{p}$, as a function of projected radius. The blue galaxies (solid squares and dotted line) have a larger $\sigma_{p}$ than the red ones (circles and solid line). The $\sigma_{p}(R)$ are fitted with $\beta=[0.0,0.5]$.

Fig. 3.- The bias function refered to the total light profile, $b_{M v}(r)$ calculated from the mass profiles derived from blue (dotted line) and red (solid line) subsamples. The upper line for both subsamples is for $\beta=0$, the lower line is for $\beta=1 / 2$.

Fig. 4.- The value of $b_{M v}$ evaluated at $r_{200}$. The blue subsample results are denoted with solid squares and the red with open circles. 


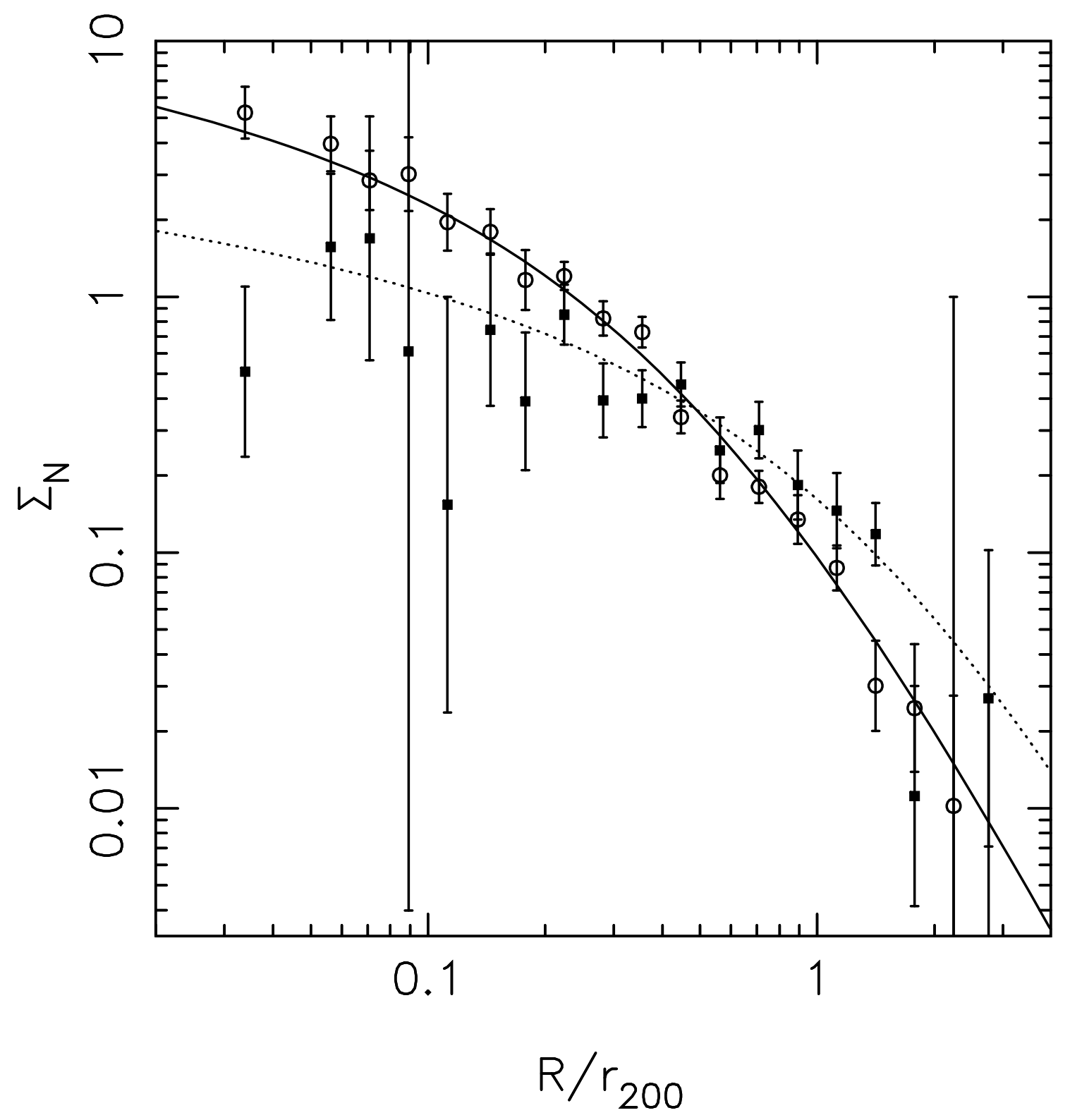

Fig. 1.- 


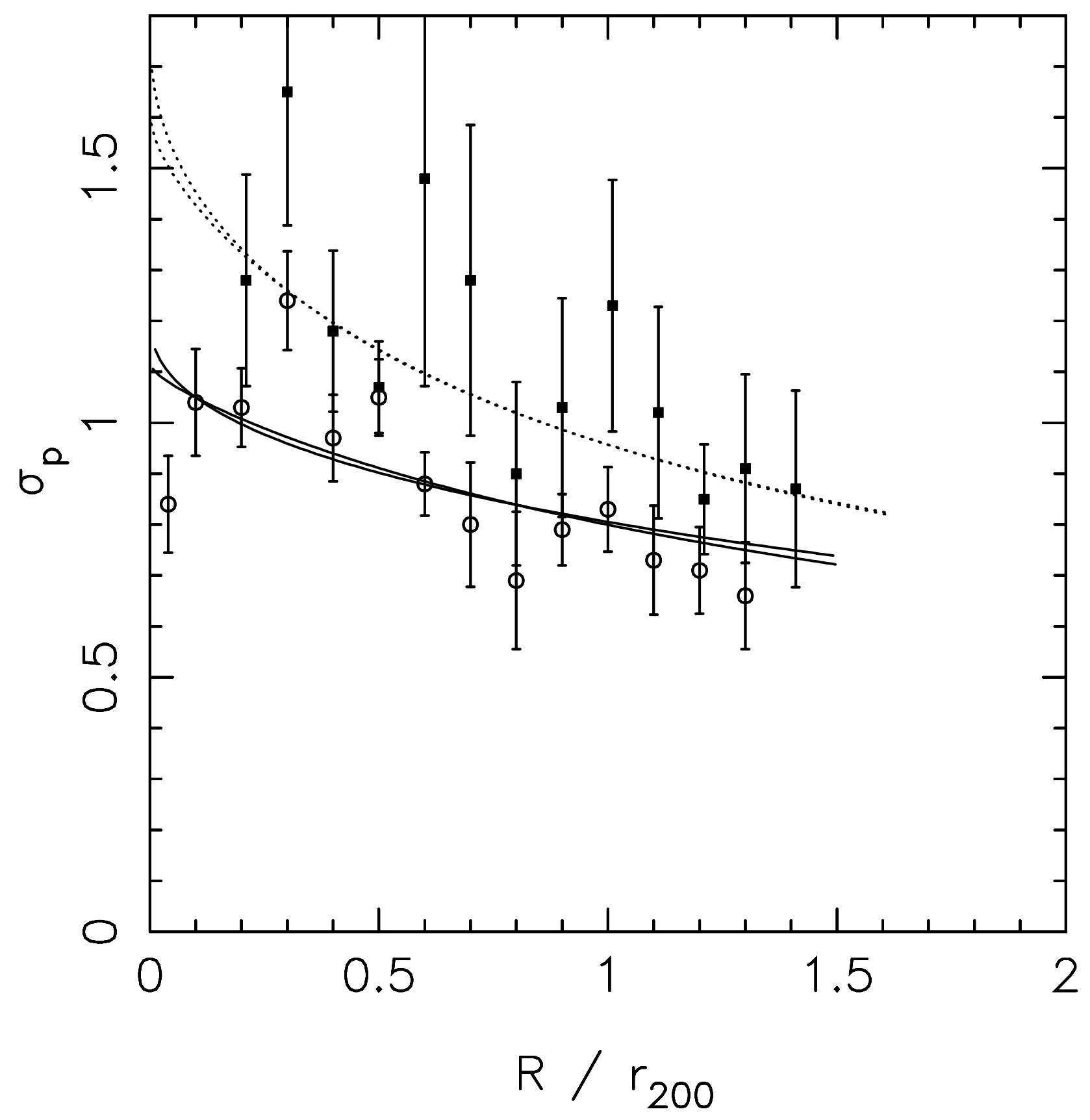

Fig. 2.- 


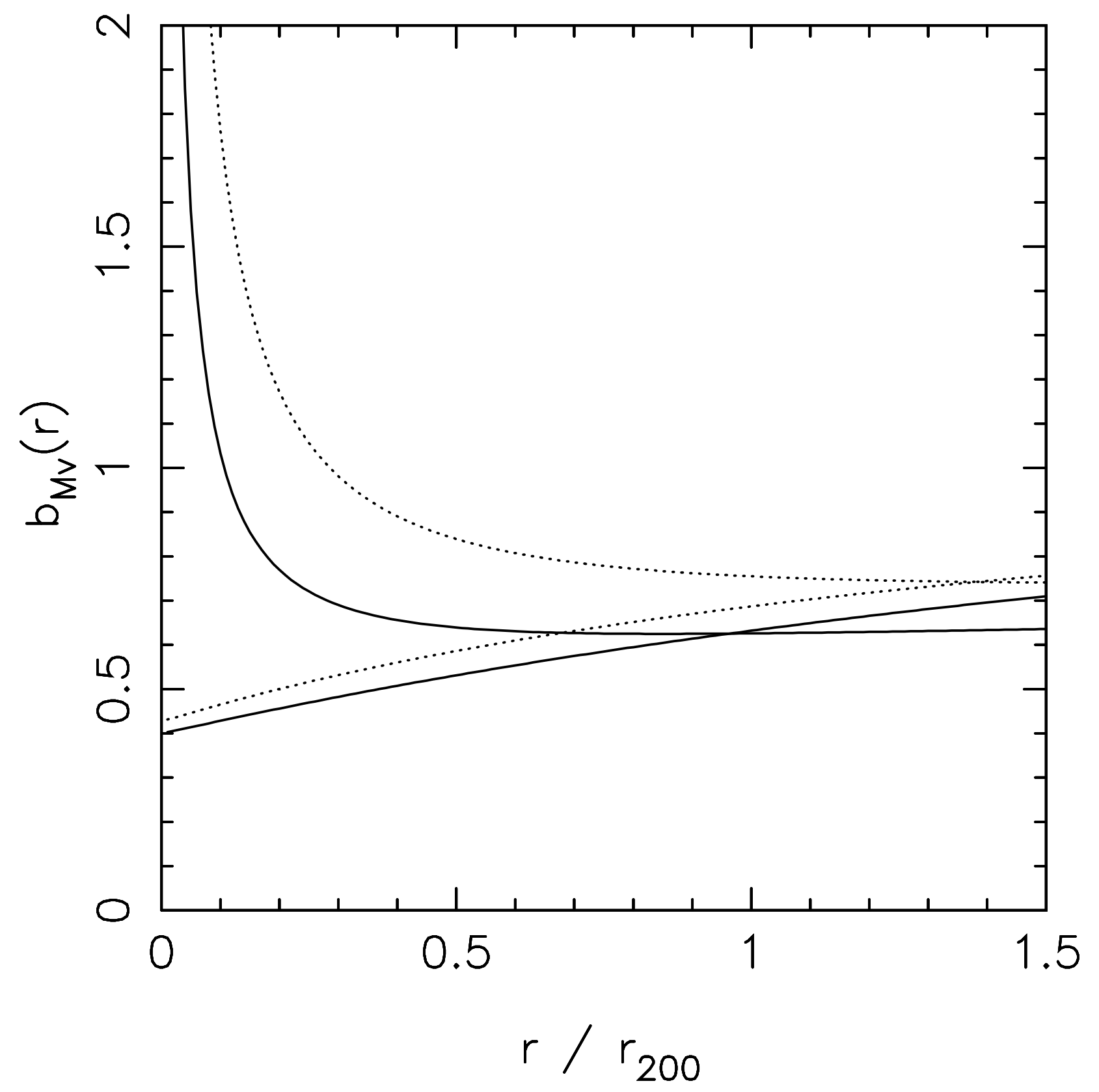

Fig. 3.- 


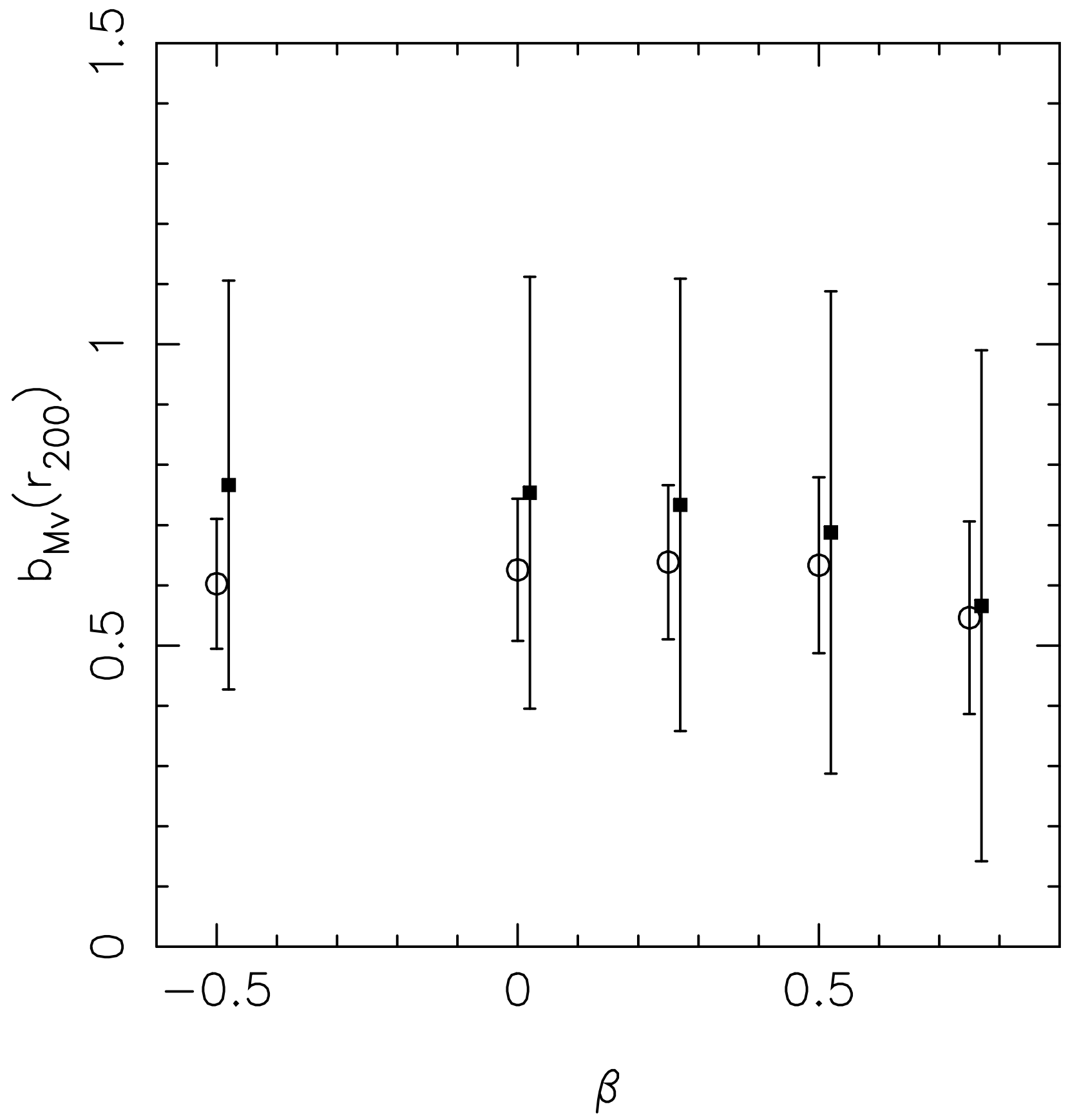

Fig. 4.- 